\title{
Analytical Solutions of the Equations One Mechanical Thermal Model of a Helical Spring NiTi for Use in Heat Engine
}

\author{
Luciana Lima Monteiro ${ }^{1}$, José Roberto da Costa Campos ${ }^{2}$, Tiago Leite Rolim ${ }^{3}$ and Ricardo Artur Sanguinetti \\ Ferreira $^{3}$ \\ 1. Academic Department of Control of Industrial Process, Instituto Federal de Educação, Ciência e Tecnologia de Pernambuco, \\ Recife 50740-540, Brazil \\ 2. Department of Mechanical Engineering, Polytechnic School of Pernambuco/UPE (University of Pernambuco), Recife 50100-010, \\ Brazil
}

3. Department of Mechanical Engineering, Federal University of Pernambuco, Recife 50670-90, Brazil

Received: May 23, 2014 / Accepted: June 05, 2014 / Published: August 25, 2014.

\begin{abstract}
Shape memory effect is capability of certain materials to recover its original shape after an apparently permanent deformation. The NiTi alloy of the composition is approximately equiatomic and it is the materials that exhibit the best characteristics for application of these properties, especially in the biomedical area, because of their excellent biocompatibility as: in the manufacture of medical and dental instruments, orthodontic wires, orthopedic materials, guide wires, stents, filters and components to realize the less invasive surgeries. In other areas, they are used for confections of electronic keys, spectacle frames, application in controllers, junction of pipes and electronic connectors among others. New research topics involving the application of these alloys super-elasticity are also known as pseudo elasticity. This event has an isothermal nature and involves the storage of potential energy in the shape memory effect and super-elasticity. In this context, this work falls within the scope of use of the technologies being an example of the work undertaken, in the course Graduate of Federal University of Pernambuco in skills in these technologies. It will present the results of a heat engine which engine element is a helical spring made of a NiTi alloy equiatomic with memory effect reversibly. The spring is triggered by a hot source $(\sim 373.15 \mathrm{~K})$ and a cold source $(273.15 \mathrm{~K})$. The machine is capable of producing a reciprocating oscillating between the two sources. Heat equations and the equations that describe the dynamic behavior of the spring were developed. Through the development of dynamic equations, it can determine the minimum mass for the motion of the machine, as well as the instantaneous and average power and overall efficiency. You can check the functionality of the machine by way of the inclination angle of the propeller and the coefficient of static friction. Among the main results, it was observed that the overall performance of the machine compared to the machines of this category showed the feasibility of the project.
\end{abstract}

Key words: Experimental mechanics, heat engine, NiTi.

\section{Introduction}

The NiTi alloys with shape memory effect are applied in heat engines. As an alternative, it can extract mechanical energy from heat sources with low temperatures as below: warm waters of industrial waste, geothermal, solar thermal energy among other.

Corresponding author: Luciana Lima Monteiro, Ph.D., professor, research field: automation and experimental mechanics. E-mail: 11_monteiro@yahoo.com.br.
Other new applications based on exactly the same principle include motors for space missions.

Alloys with shape memory effect have been considered as candidates for medium operations applied in heat engines, because of the phase transformation which occurs during the compression and expansion operations. In spite of thermodynamic irreversibility, the crystallographic reversibility $\beta \rightarrow$ $\mathrm{M} \rightarrow \beta$ makes the shape memory effect as a great 
attraction for the manufacture of heat machines, because it has the efficiency that comes closest the efficiency of the Carnot machine [1].

This paper shows a mechanical thermal model of a heat machine that uses as motor element, a helical spring made of alloy equiatomic NiTi shape memory may affect reversible. An experimental prototype was built to assess the dynamics of the spring as the equations that describe its motion were also developed.

The paper is organized as follows: Section 2 shows, the heat engine; Section 3 shows the principle of operation of the heat engine; Section 4 shows spring training; Section 5 develops the equations of the behavior of the spring, Sections 5.1 shows the dynamic equations of spring, Sections 5.2 presents equations of motion (passive) axial, Sections 5.3 describes equations of motion (active) axial and Sections 5.4 shows equations of the angular motion; Section 6 presents results and discussions; Section 7 determines the instantaneous and average power; and Section 8 gives conclusions.

\section{The Heat Engine}

The heat engine consists basically of a rigid PVC (polyvinyl chloride) cylinder with a diameter of 1.66", a piece of aluminum, pin guide and spring NiTi. This cylinder made a helical groove with a width of 0.24 ", made in its side surface, a sweeping angle of $180^{\circ}$. A piece of aluminum located in the upper part of the cylinder is fixed to the cylinder by bolts on its side, this piece has the same length as the cylinder, and serves to support the spring during its movement. This piece of aluminum has a circular slot which moves the guide pin of the spring. This guide pin is fixed on one end of the spring NiTi and the other side was fixed to a nylon wire, with $1 \mathrm{~mm}$ diameter pulling load. Also as part of the whole machine, there are two reservoirs that the spring dipped. A reservoir has hot water with constant temperature to $373.15 \mathrm{~K}$ and the other cold water reservoir has a constant temperature of $273.15 \mathrm{~K}$.

Fig. 1 presents the heat engine with its main parts described.

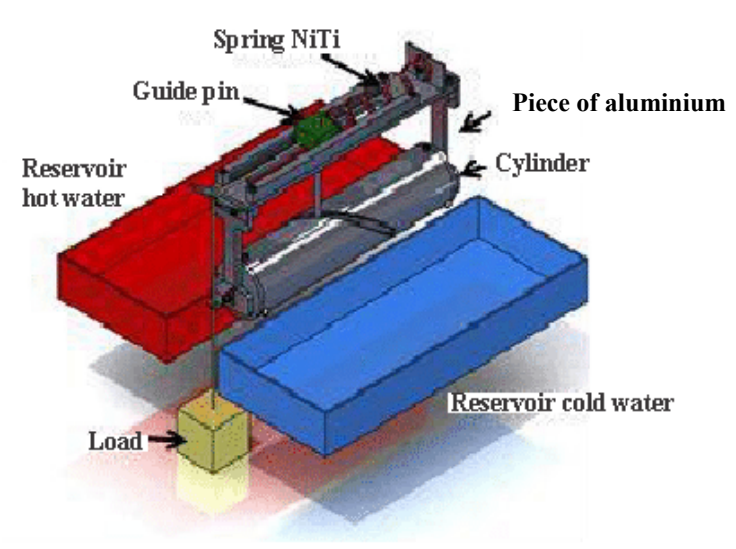

Fig. 1 Heat engine.

\section{Principle of Operation of the Heat Engine}

The NiTi coil spring positioned as shown in Fig. 1, it will move due to two factors: the memory effect phenomenon, or "two way" which is one of the properties of martensitic transformations thermoelastic. Water tanks contacts hot and cold water, and efforts made by the spring on the helical groove, due to the movement of the load fixed on the nylon wire and the helical spring NiTi. The movement of the spring is helical with an angle approximately $180^{\circ}$ and covered the slot of the cylinder through guide pin between the two reservoirs. The cycle begins when the spring is extended in the martensitic phase under the action of a load; as it operating, the cold water reservoir is with constant temperature at $273.15 \mathrm{~K}$, walks the helical groove by the guide pin. By dipping in hot water reservoir, it reaches to $373.15 \mathrm{~K}$ (warm source) above the $A_{f}$ temperature (final temperature of austenitic transformation). And so it is generating a continuous reciprocating motion.

The aluminum piece in the upper part of the cylinder moves with the spring because the guide pin is pivoted with the piece of aluminum. When the spring is in the cold water reservoir, the spring is stretched and the loading fix on the nylon wire descend and when the spring is in the hot water reservoir, the spring contracts and the load attached to the nylon wire, thereby, it perform a reciprocating movement to produce a continuous and mechanical work. In Fig. 2, a simplified form of the behavior of the spring is shown. 


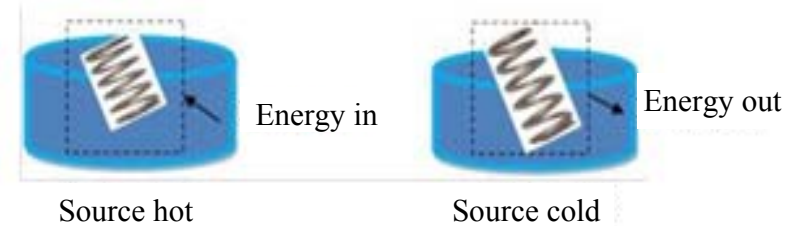

Fig. 2 Behavior of movement of spring.

\section{Obtaining and Spring Training}

To get the shape memory effect reversibly in spring $\mathrm{NiTi}$, it was need to be submitted to training. The shape memory effect reversible happens when the change of shape is promoted only by temperature variation. During training or education process, it can associate a shape to each phase as a function of a given thermomechanical treatment [2]. For training we used a spring direct current source to simulate the heating being applied a current of $12 \mathrm{~A}$ at a voltage of $4.4 \mathrm{~V}$. By using the cooling spring, the air in the laboratory ambient temperature is around $295.15 \mathrm{~K}$. in order to obtaining the movement of contraction and distension of the spring, we used standardized masses to fix on your end. Table 1 shows the results of varying of the mass and the respective distention of the spring.

\section{Equations of the Behavior of the Spring}

To develop equations reflects the behavior of the spring with respect to temperature; we should consider the region of phase transformation and mechanical properties of the spring. The spring movement was described by ordinary differential equations based on Newton's Second Law [3]. We can apply some simplifying assumptions and considerations in the development of equations [4] and in basics of thermodynamics, conservation of energy and heat transfer [5]. It is considered only in heat exchange between the spring and the reservoir water. Considering an energy balance in the control volume, Eq. (1) describes the behavior of the spring when entering the hot water reservoir:

$$
m c \frac{\mathrm{d} T}{\mathrm{~d} t}=h A\left(T_{A q}-T_{\text {spring }}\right)
$$

Table 1 Displacement of the spring.

\begin{tabular}{ll}
\hline Mass $(\mathrm{g})$ & Displacement $(\mathrm{cm})$ \\
\hline 1,950 & 17.5 \\
2,207 & 16.5 \\
2,407 & 15.5 \\
2,707 & 15.0 \\
\hline
\end{tabular}

where, data were measured experimentally as: $m$ : mass spring (12.3367 g); TAq: temperature of hot water $(373.15 \mathrm{~K})$; and A: Area Spring $(12,377.88$ $\left.\mathrm{mm}^{2}\right)$.

The specific heat $c$ and $h$ convection coefficient tables were obtained from the literature, respectively, $322 \mathrm{j} / \mathrm{Kg} / \mathrm{K}$ and $1,000 \mathrm{~W} / \mathrm{m}^{2} / \mathrm{K}$ [5]. The value of $h$ is not definitive and may improve [6].

Heating occurs in the spring of the transformation of $\mathrm{M}$ (martensite phase) to A (austenite phase). On cooling, the transformation of $\mathrm{A}$ (austenite phas e) in spring to the $M$ (martensite phase) occurs. Eq. (2) describes phase transformation as a function of temperature [7].

$$
\begin{gathered}
\xi=\frac{\xi_{m}}{1+\operatorname{esp}\left[\frac{6.2}{A_{f}-A_{s}}\left(T_{\text {spring }}-\frac{A_{s}-A_{f}}{2}\right)\right]} \text { (Heating) } \\
\xi=\frac{1-\xi_{a}}{1+\operatorname{esp}\left[\frac{6.2}{M_{f}-M_{s}}\left(T_{\text {mola }}-\frac{M_{s}-M_{f}}{2}\right)\right]}(\text { Cooling })
\end{gathered}
$$

$A_{s}$ and $A_{f}$ are the initial and final austenitic transformation temperatures respectively, $M_{s}$ and $M_{f}$ are the initial and final martensitic transformation temperatures respectively. $\xi$ is the fraction transformed of martensite ( $0-1$ or $0-100 \%), \xi_{m}$ is the larger fraction of martensite during cooling process (or initial value during heating), $\xi_{a}$ is the initial value of the fraction of austenite during heating process (or initial value during cooling).

In Fig. 3, there is a behavior that occurs in alloys with shape memory effect.

According to Ref. [8], the equations of the mechanical properties of shape memory alloy are obtained from a model of layers in the phase austenite (high temperature), the spring becomes elastic and thus for $\xi=0$, the relationship between stress and strain is given in Eq. (3): 

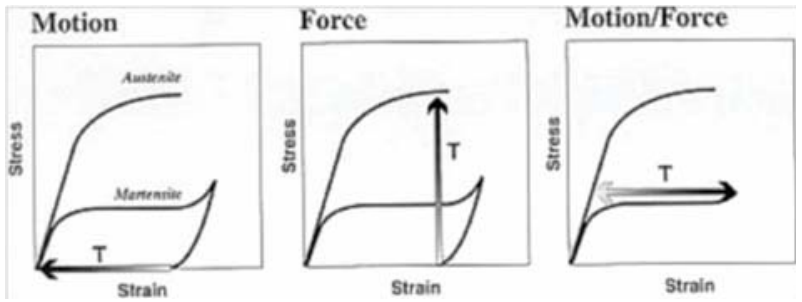

Fig. 3 The shape memory effect in stress-strain curve. Source: Gilbertson; Miranda, 1992.

$$
\sigma_{A}=E_{A} \varepsilon
$$

where, $\sigma_{A}$ is the voltage at the austenite phase; $E_{A}$ is the elastic modulus (Young's modulus) of austenite and $\varepsilon$ is the strain.

In the martensite phase $\xi=1$, this phase is plastic and spring behaves describes according to Eq. (4):

$$
\sigma_{M}=E_{m} \varepsilon \text { if }|\varepsilon| \leq\left|\varepsilon_{m y}\right|, \sigma_{m}=E_{m} \varepsilon_{m y} \text { if }|\varepsilon|>
$$

where, $\sigma_{M}$ : maximum tension of the spring in martensite phase; $E_{m}$ : modulus of the elasticity in the martensite phase; and $\varepsilon_{m y}$ : elastic limit in martensite phase.

The characteristic graph representing this equation is shown in Fig. 4.

In the intermediate phase $(0<\xi<1)$, the relationship between stress and strain is given in Eq.

$$
\begin{array}{r}
\sigma=\left[\xi \sigma_{M}+(1-\xi) \sigma_{A}\right] \rightarrow \varepsilon=\frac{\sigma}{\xi E_{m}+(1-\xi) E_{A}} \text { to }|\varepsilon| \leq \\
\left|\varepsilon_{m y}\right| \text { e } \varepsilon=\frac{\sigma-\xi E_{m} \varepsilon_{m y}}{(1-\xi) E_{A}} \text { to }|\varepsilon|>\left|\varepsilon_{m y}\right|
\end{array}
$$

Eq. (6) provides the stress of the spring based on Eqs. (3)-(5):

$$
\frac{F_{\text {spring }}}{A_{\text {cross }}}=\left[\xi E_{M}+(1-\xi) E_{A}\right] \frac{\Delta l}{l_{0}}
$$

where, $A_{\text {cross: }}$ : cross-sectional area of the wire (3.8 $\left.\mathrm{mm}^{2}\right) ; \Delta l$ : linear variation of the spring $(175 \mathrm{~mm})$; and $l_{0}$ : initial length of the spring $(73 \mathrm{~mm})$.

\subsection{Dynamic Equations of Spring}

The development of differential equations that

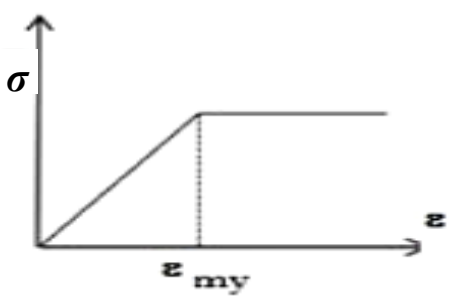

Fig. 4 Characteristic graph of stress-strain in the martensite phase.

describe the movement of the spring was considered, because the symmetry of motion, gravitational effects are negligible, since the variation of potential energy is zero over a cycle. The spring move through the slot of the cylinder, through the guide pin, it will develops a resultant reaction force. This resultant force produced by the shape memory effect is decomposed into two components: one axial and one radial. Eq. (7) describes the resultant force.

$$
F_{R}=F_{\text {active }}-\text { load }-F_{\text {friction }}
$$

Eq. (7) shows the force resulting from axial active movement, contraction of the spring. And Eq. (8) represents the force resulting from axial passive movement, stretching the spring:

$$
F_{R}=\text { load }-F_{\text {passive }}-F_{\text {friction }}
$$

\subsection{Equations of Motion (Passive) Axial}

Fig. 5 shows a schematic drawing of rigid PVC cylinder and groove with the decomposition of the forces and load representation. The equations is considered: The origin of the coordinate $\mathrm{x}$ is the time of the start of the slot; The $\mathrm{x}$ coordinate coincides with the axis of the cylinder and the force tangent to the groove $R_{M}$ is the generic point. Passive $F_{M}$ is spring force in martensitic phase (expansion). $F_{M}$ is the axial component of $R_{M}$. And $F_{T}$ is the tangential component of the $R_{M} . L$ is slot length. $X$ is half the slot length. And $P$ is the weight force where $m$ is the mass of the load. By determining the angle of the helix $(\alpha)$, one can obtain a relation between the axial and tangential forces. As shown in Fig. 5, the inclination angle of the helix is formed by the line of the propeller and 


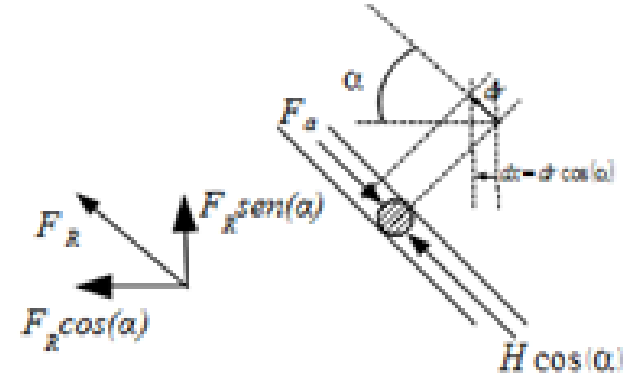

Fig. 5 Resultant force along the slot (considering $F_{x}=H$ ). horizontal direction.

Eq. (9) described the calculation of the angle of inclination:

$$
\tan \alpha=\text { Cpitch of the helix }
$$

where, $C$ is the circumference of the cylinder, $C=$ $2 \cdot \pi \cdot r$. Then we substitute it in Eq. (9): $\tan \alpha=2 \cdot \pi \cdot r \cdot \mathrm{L}$. Considering the position of the guide pin in the position of the center of the diameter then: $\tan \alpha=$ $(\pi \cdot r) / x$. Eq. (10) considers the triangle of forces in Fig. 5 .

$$
\tan \alpha=\frac{F_{r}}{F_{M}}
$$

And the $R_{M}$ is friction force, i.e., a force opposing the movement of the spring. And we can apply the second law of Newton in the spring to deduce Eq. (11). Eq. (11) is the equation of motion (passive) axial.

$$
M \frac{\mathrm{d}^{2} x(t)}{\mathrm{d} t^{2}}+b \frac{\mathrm{d} x(t)}{\mathrm{d} t}+k x(t)=m g
$$

where, $M$ is the mass of the guide pin coupled with the mass of the spring $b((\mathrm{dx}(\mathrm{t})) ; \mathrm{d} t$ is the force of resistance to axial movement, i.e., it is a viscous friction force, being proportional to the velocity.

\subsection{Equation (Active) Axial Movement}

Fig. 6 has the same considerations of Fig. $5 ; f_{a}$ is the active force of the spring in the austenitic phase (contraction). We should consider the same development done to the passive movement and apply Newton's second law in the spring results in Eq. (12). Eq. (12) is the axial movement of the spring.

$$
M \frac{\mathrm{d}^{2} x(t)}{\mathrm{d} t^{2}}+b \frac{\mathrm{d} x(t)}{\mathrm{d} t}-k x(t)=-m g
$$

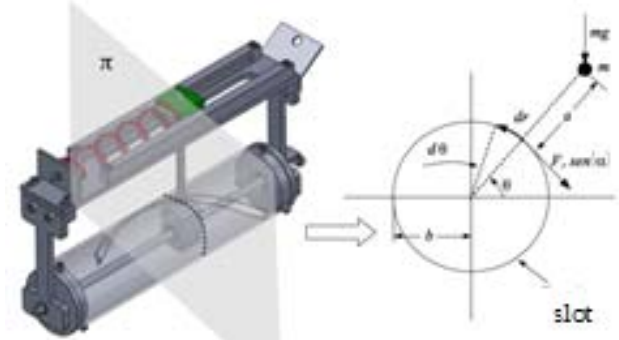

(a)

(b)

Fig. 6 Angular movement of the spring.

\subsection{Equations of the Angular Movement}

It is found when the spring is moving around, the slot cylinder is stretching and contacting the reservoirs, in this way, it approaches a linear behavior. At the same time, the spring runs approximately $180^{\circ}$ to perform the reciprocating movement.

Fig. 7 shows detail of the angular movement. The tangential efforts system is considered: $F_{T}$ is tangential force; $F_{R}$ is resistant tangential force; $r$ is the radius of the cylinder and $\theta$ is the angular coordinate, we can apply Newton's Second Law of the spring to deduce Eq. (13).

Eq. (13) describes the tangential movement of the spring:

$$
M(t) \mathrm{d} t+b(t) \mathrm{d} t-k \theta(t)=0
$$

\section{Results and Discussion}

The analytical solutions of the equations of mechanical and thermal model referred were presented.

The development of the analytical solution of the heat equations are necessary to determine the thermal behavior of the spring, when immersed in the reservoirs is described in Eqs. (14)-(17).

$$
\begin{gathered}
m C_{p} \frac{\mathrm{d} T}{\mathrm{~d} t}=h A\left(T_{f Q}-T\right) \text { in which } \frac{\mathrm{d} T}{\mathrm{~d} t}=\frac{h A T_{f Q}}{m C_{P}}- \\
\frac{h A T}{m C_{P}} \text { and } \frac{\mathrm{d} T}{\mathrm{~d} t}+\left(\frac{h A}{m C_{P}}\right) T=\frac{h A T_{f Q}}{m C_{P}}
\end{gathered}
$$

Eq. (15) is a linear differential equation of 1 st order with solution $T$ where $T=f(t)$. The equation is of the type: 


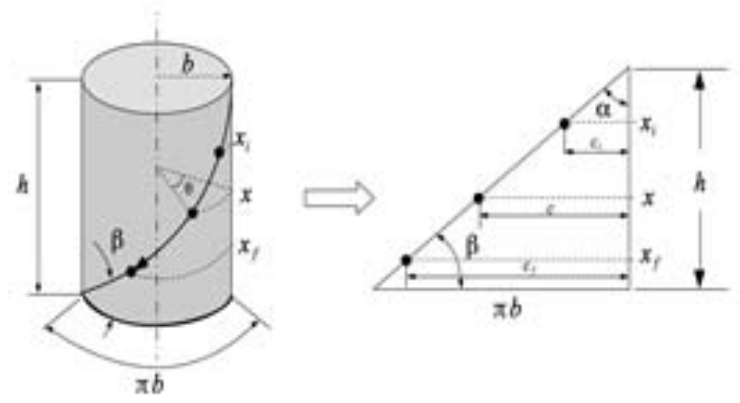

Fig. 7 Detail of the angular movement.

$$
\begin{gathered}
\frac{\mathrm{d} T}{\mathrm{~d} t}+a^{\prime} T=b^{\prime} \\
\text { in which } a^{\prime}=\frac{h A}{m C_{P}} \text { and } b^{\prime}=\frac{h A T_{f Q}}{m C_{P}}
\end{gathered}
$$

To determine the solution to Eq. (15), first, find the integrating factor $\mu(t)$

$$
\mu(t)=e^{\int a^{\prime}} \mathrm{d} t
$$

by multiplying the integrating factor then:

$$
e^{a^{\prime} t} \frac{\mathrm{d} T}{\mathrm{~d} t}+e^{a^{\prime} t} a T=e^{a^{\prime} t} b^{\prime}
$$

The left side of Eq. (16) is the derivative of a product, and it can then be rewritten as:

$$
\frac{\mathrm{d}\left(e^{a^{\prime} t} T\right)}{\mathrm{d} t}=e^{a^{\prime} t} b^{\prime}
$$

We integrate these equations to deduce that:

$$
\begin{gathered}
u=a^{\prime} t \rightarrow \mathrm{d} u=a^{\prime} \mathrm{d} t \rightarrow \mathrm{d} t=\frac{\mathrm{d} u}{a^{\prime}} \\
\int \frac{\mathrm{d}\left(e^{a^{\prime} t} T\right)}{\mathrm{d} t} \mathrm{~d} t=\frac{b^{\prime}}{a^{\prime}} \int e^{u} \mathrm{~d} u \\
e^{a^{\prime t}} T(t)=\frac{b^{\prime}}{a^{\prime}} e^{a^{\prime} t}+k^{\prime}
\end{gathered}
$$

Resulting in

$$
T(t)=\frac{b^{\prime}}{a^{\prime}}+k^{\prime} e^{-a^{\prime} t} \text { in which } k^{\prime}=273-\frac{b^{\prime}}{a^{\prime}}
$$

Apply the boundary conditions for: $t=0 \mathrm{~s}, T=$ $273 \mathrm{~K}$, so find the value of $k^{\prime}$ in Eq. (17).

We can substitute the values of $a^{\prime}, b^{\prime}, e$ and $k^{\prime}$ to deduce Eq. (18).

Eq. (18) is the analytical solution of spring behavior during heating. Eq. (18) is determined by the time that the spring attains the temperature of the hot source.

$$
T(t)=T_{f Q}\left(1-e^{-\left(\frac{h A}{m C_{P}}\right) t}\right)+273 e^{-\left(\frac{h A}{m C_{P}}\right) t}
$$

Eq. (18) shows the behavior of the spring. The data used were: $h=1,000 \mathrm{~W} /\left(\mathrm{m}^{2} \mathrm{~K}\right) ; A=2.9283 \cdot \mathrm{x} \cdot 10^{-3}$ $\mathrm{m}^{2} ; m=0.01213 \mathrm{~kg} ; C_{p}=200 \mathrm{~J} / \mathrm{kg} / \mathrm{K} ;$ and $T_{f 0}=$ $373.15 \mathrm{~K}$.

Fig. 8 represents the behavior of the spring during heating, so it can observed rapid heating immediately, when the spring comes into contact with the hot source.

In less than $5 \mathrm{~s}$ spring attains $373 \mathrm{~K}$ which is the temperature of the hot source, noting that the spring arrives at the hot water reservoir at a temperature of $273 \mathrm{~K}$. To determine the behavior of the spring when immersed in cold source, analytical development is the same used for the heating of the spring, since only the initial conditions change, it is considered that the spring arrives at the cold source at temperature of $373.15 \mathrm{~K}$. We can apply the boundary conditions for: $t=0 \mathrm{~s}, T=373.15 \mathrm{~K}$, so we can find the value of $k$.

$$
k^{\prime}=373-\frac{b^{\prime}}{a^{\prime}}
$$

We can substitute the values of $a^{\prime}, b^{\prime}, e$ and $k^{\prime}$ to deduce Eqs. (19) and (20).

Eq. (20) is the analytical solution of spring behavior during cooling.

Eq. (20) is determined by the time that the spring attains the temperature of the cold source.

Fig. 9 represents the behavior of the spring during cooling.

$$
T(t)=\frac{b^{\prime}}{a^{\prime}}\left(1-e^{-^{a^{\prime} t}}\right)+373 e^{-^{a^{\prime} t}}
$$

$$
T(t)=T_{f F}\left(1-e^{-\left(\frac{h A}{m C_{P}}\right) t}\right)+373 e^{-\left(\frac{h A}{m C_{P}}\right) t}
$$

The equations that describe the axial movement and angular movementof the spring are linear differential equations that we can obtain analytical solutions. It is important to rewrite them in the canonical way, as follows in Eq. (21). 


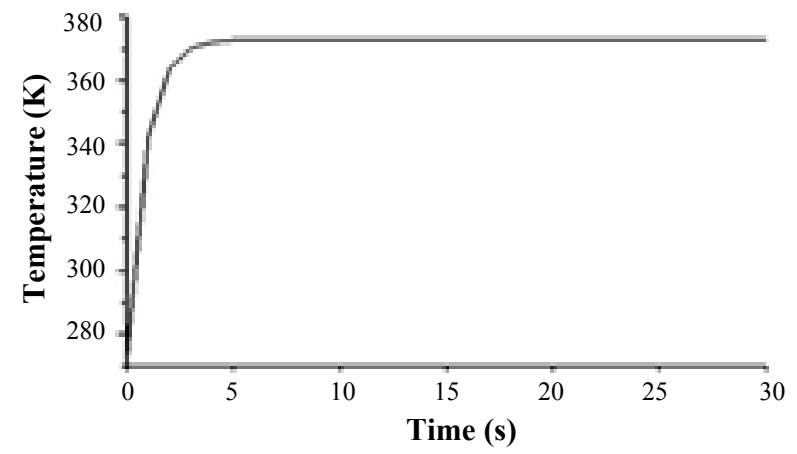

Fig. 8 Spring behavior during heating.

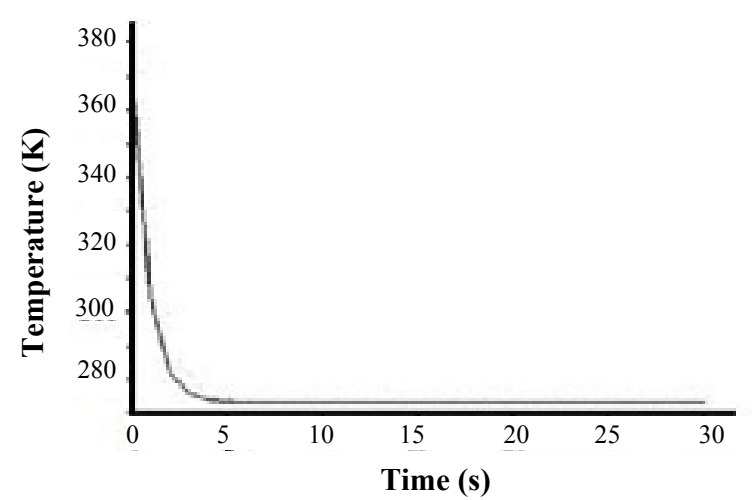

Fig. 9 Spring behavior during cooling.

$$
\begin{gathered}
{\left[k\left(x-x_{0}\right)-T_{m}\right]\left[\cos ^{2}(\alpha)-\mu \sin (\alpha) \cos (\alpha)\right]=} \\
M \frac{\mathrm{d}^{2} x}{\mathrm{~d} t^{2}}
\end{gathered}
$$

We can develop Eq. (21):

$$
\begin{gathered}
M \frac{\mathrm{d}^{2} x}{\mathrm{~d} t^{2}}=T_{m} c_{x \mu}+k c_{x \mu} x-k c_{x \mu} x_{0} \\
\frac{\mathrm{d}^{2} x}{\mathrm{~d} t^{2}}-\left(\frac{k c_{x \mu}}{M}\right) x+\left(\frac{k c_{x \mu} x_{0}+T_{m} c_{x \mu}}{M}\right)=0
\end{gathered}
$$

We can rewrite Eq. (21) in the canonical form:

$$
\frac{\mathrm{d}^{2} x}{\mathrm{~d} t^{2}}-a^{\prime \prime} x+b^{\prime \prime}=0
$$

where,

$$
\begin{gathered}
a^{\prime \prime}=-\frac{k c_{x \mu}}{M} \\
b^{\prime \prime}=\frac{k c_{x \mu} x_{0}+T_{m} c_{x \mu}}{M}
\end{gathered}
$$

Eq. (22) describes the angular movement of the spring.

$$
\frac{\mathrm{d}^{2} \theta}{\mathrm{d} t^{2}}=\left[\frac{h \sin (\alpha) b k c_{\mu}}{\pi M(a+b)^{2}}\right] \theta-\frac{\left(k x_{0}+T_{m}\right) \sin (\alpha) b c_{\mu}}{M(a+b)^{2}}
$$

The determination of solutions of Eq. (22) was done by using Laplace transformation method and we can deduce Eqs. (23) and (24).

$$
\begin{aligned}
& \theta(t)=\frac{d^{\prime \prime}}{c^{\prime \prime}}\left[\cos \left(\sqrt{c^{\prime \prime}} t\right)-1\right] \\
& x(t)=\frac{b^{\prime \prime}}{a^{\prime \prime}}\left[\cos \left(\sqrt{a^{\prime \prime}} t\right)-1\right]
\end{aligned}
$$

We can enter the following information in Eqs. (23) and (24):

$k$ : spring constant, experimentally measured 0.109 $\mathrm{N} / \mathrm{mm}$;

$\alpha$ : angle of inclination, $33^{\circ}$;

$M$ : mass of the spring, $0.604 \mathrm{~kg}$;

$T_{m}$ : tension, $7.33 \mathrm{~N}$;

$X_{0}$ : length of spring, $73 \mathrm{~mm}$;

$b$ : cylinder radius, $31.75 \mathrm{~mm}$;

$h$ : half the pitch of the helicoids, $75 \mathrm{~mm}$;

$(a+b)$ : distance of the $M$ until the $0147.75 \mathrm{~mm}$;

$\mu$ : coefficient of friction, 0.61[9].

Figs. 10-12 check the axial and angular displacement of the spring. In angular movement, the program plotted the equation solution in the range between $\theta=0$ and $\theta=\pi$. The program simulated the actual movement of the spring starting from hot source to cold source. The axial movement is observed that the spring is stretched from its original length $30 \mathrm{~mm}$. The spring moves from the hot source to the cold source will take $25 \mathrm{~s}$. Yet it can be seen that the acceleration of the spring is very slow to start movement.

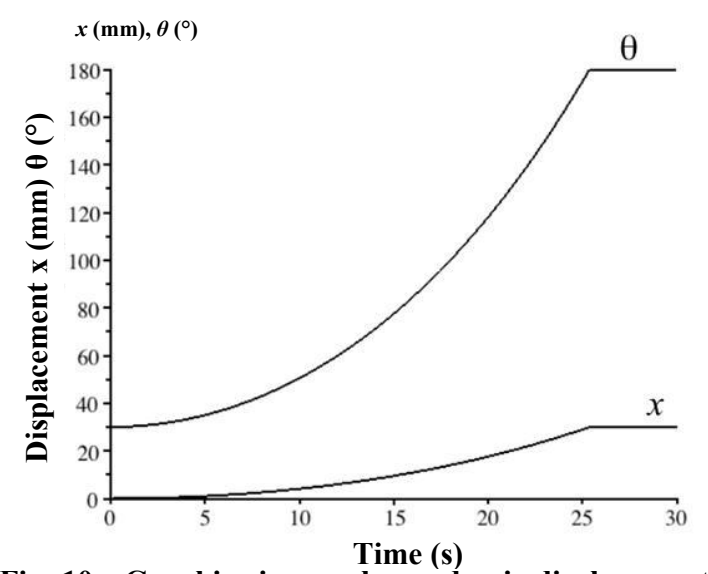

Fig. 10 Graphics in angular and axis displacement of the spring. 


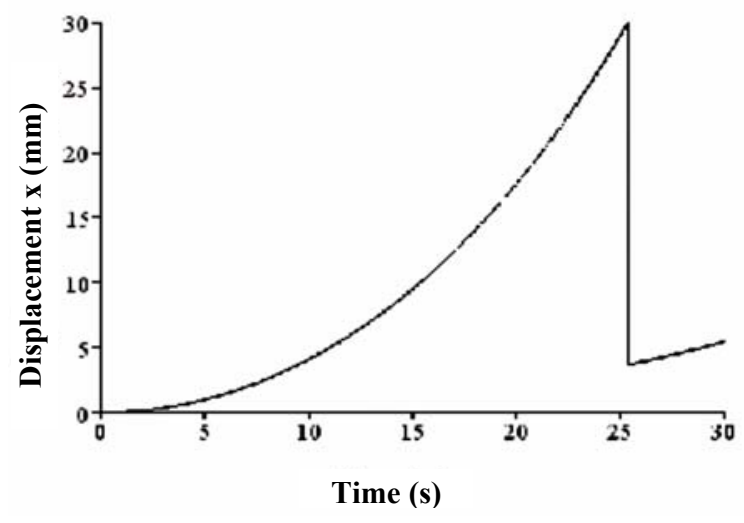

Fig. 11 Graphics in axis displacement of the spring.

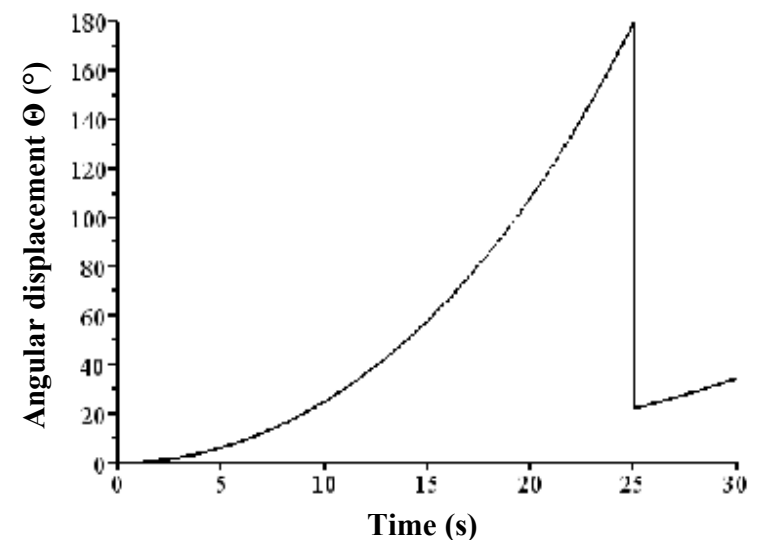

Fig. 12 Graphics in angular displacement of the spring.

\section{Determination of the Instantaneous and Average Power}

Figs. 13-15 show the graphs of the instantaneous and average power, respectively.

The determined value was $0.5 \mathrm{~W}$ for the power. The results presented in the graphs above are satisfactory with the reality of this type of heat engines. Cornick and Palu [10] states that the power of a heat engine

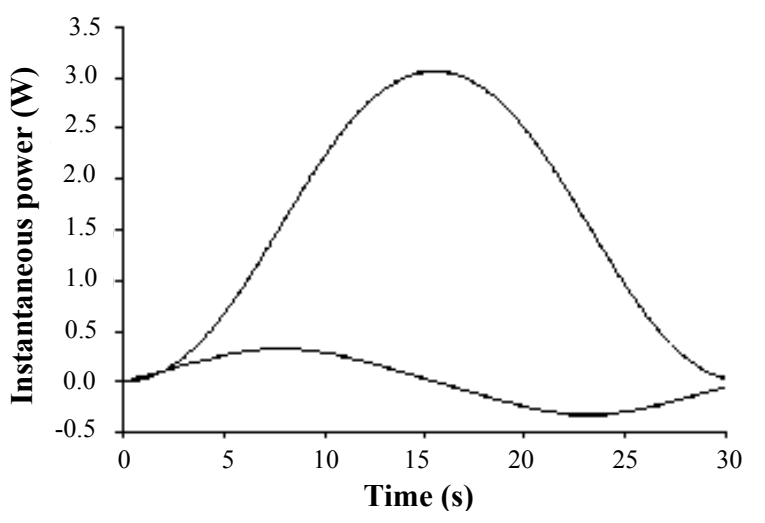

Fig. 13 Graphics of power of the spring.

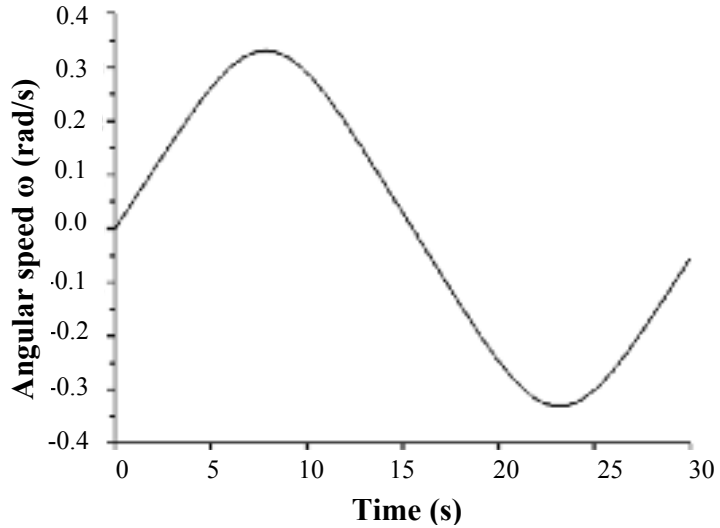

Fig. 14 Graphics of angular speed of the spring.

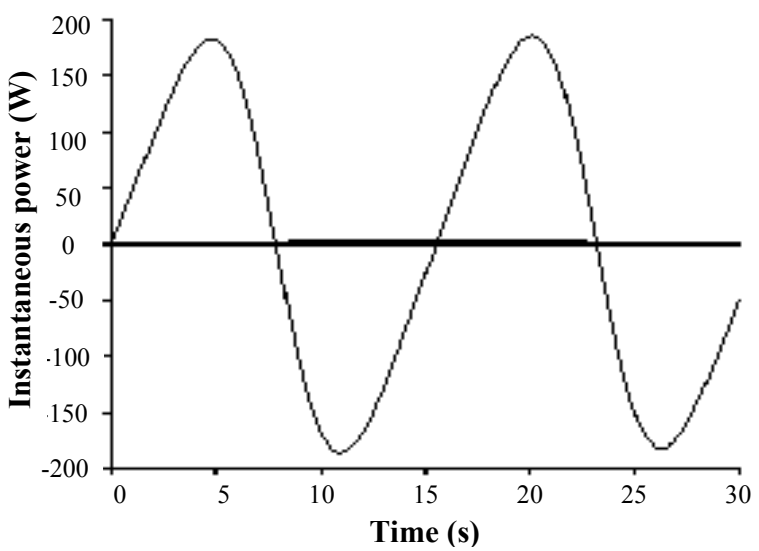

Fig. 15 Graphics of instantaneous power of the spring.

with shape memory alloy is a function of the rate of heat transfer between the active element and the temperature of the reservoirs.

\section{Conclusions}

The machine model works only for values of the coefficient of friction which satisfy the relation: $\tan ^{-1}(\alpha) \geq \mu_{e}$.

The powers dissipated in the axial motion and angular motion of the spring was determined experimentally and $7.33 \mathrm{~N}$ and $7 \mathrm{~N}$ were found respectively. The complete machine cycle is developed at a time (period) of $25 \mathrm{~s}$. The thermal model was designed despite of the simplifying considerations, though, it is not the models of the phase transformations (coupled), and was reliable for forecasting the behavior of the spring.

The thermal inertia of the machine movement (mechanical inertia) increases during the cycle. Even 
there are uncoupled mechanical and thermal studies, as is done in most developed mathematical models for simulating movements, the results was proved satisfactorily. The simulation of the movement of the spring make a commercial software educational version and it describes the actual movement of the machine.

\section{References}

[1] Salzbrenner, R. 1984. "Shape Memory Heat Engines." Journal of Materials Science 19: 1827-35.

[2] Lagoudas, D. C. 2008. Shape Memory Alloys Modeling and Engineering Applications. New York: Springer Science+Business Media.

[3] Singerisu, R.. 2009. Mechanical Vibrations. USA: Pearson.

[4] Alexandra, L., Grant, D., and Hayward, V. 1997. "Design and Comparison of High Strain Shape Memory Alloy Actuators." In Proceeding of International Conference on
Robotic and Automation, 260-65.

[5] Incropera, F. P., Dewitt, D. P., Bergman, T. L., and Lavine, A. S. 2008. Fundamentals of Heat Transfer and Mass. USA: Wiley.

[6] Wakjira, J. 2001. "The VT1 Shape Memory Alloy Heat Engine Design." master thesis, Polytech Institute and State University.

[7] Ikuta, K., Tsukamoto, M., and Hirose, S. 1991. "Mathematical Model and Experimental Verification of Shape Memory Alloy for Designing Micro Actuator." In Proceeding of the IEEE on Micro Electromechanical Systems, an Investigation of Microstructure, 103-8.

[8] Romano, R., and Tannuri, E. A. 2008. "Modeling, Control and Experimental Validation of a Novel Actuator Based on Shape Memory Alloy." Mechatronics 19: 1169-77.

[9] Kazuhiro, O., and Marvin, W. C. 1998. Shape Memory Materials. Cambridge: Cambridge University Press.

[10] Cornick, M., and Paul, G. 1986. "Shape Memory Effect Heat Engine Performance.” Appllied Energy 24: 221-43. 